K.A. van der Hucht, G. Koenigsberger \& P.R.J. Eenens, eds.

\title{
Observations of colliding-wind effects at long wavelengths: IR to radio
}

\author{
Peredur M. Williams \\ Royal Observatory, Blackford Hill, Edinburgh EH9 3HJ, UK
}

\begin{abstract}
The supersonic winds of the most luminous stars carry kinetic energy as well as mass. Where such stars are members of binary systems, some of this energy is released in strong shocks which form on both sides of the windcollision boundary. Originally, the consequent heating of the gas in such systems was predicted to make the stars strong X-ray sources. However, the most conspicuous colliding-wind effects have been observed at longer wavelengths: synchrotron radiation from the shock-accelerated electrons and, most unexpectedly, infrared emission from dust condensing in the shock-compressed gas. Recent developments in observational techniques, especially increased spatial resolution, have led to delineation of these effects, and extensive observing campaigns have given a better idea of their incidence.
\end{abstract}

\section{Introduction: appreciation of colliding-wind (CW) effects}

The dense, supersonic winds that give WR stars their characteristic spectra carry significant mass $\left(\sim 3 \times 10^{-5} \mathrm{M}_{\odot} \mathrm{y}^{-1}\right)$ and kinetic energy $\left(\sim 10^{4} \mathrm{~L}_{\odot}\right)$. In their study of X-ray production from binaries, Prilutskii \& Usov (1976) recognized that collision of a WR stellar wind with that from a companion OB star could be a significantly stronger source of X-rays than collision of the WR wind with the OB star itself. WR binaries were, indeed, observed to be stronger X-ray sources than single WR stars (Pollock 1987) and colliding wind structures have been modelled e.g., analytically by Usov (1992) and numerically by Stevens $e t$ al. (1992). At longer wavelengths, the observations came first. A correlation between X-ray activity and radio brightness amongst OB and WR stars was noted by Chlebowski (1989). Williams et al. (1990a) deduced from its systematic variation that the non-thermal radio flux from the $\mathrm{WC} 7+\mathrm{O} 4-5$ binary WR 140 (HD 193793) was coming from a source moving along with the O4-5 star in its orbit through the wind of the WC7 star. The same binary shows episodes of circumstellar dust formation in phase with the orbit (Williams et al. 1990), and Usov (1991) has shown how the high densities $\left(10^{3-4}\right.$ times that of the undisturbed wind) required for this to occur can be produced in colliding-wind shocks. Particle acceleration and the generation of non-thermal radio emission in WR+OB wind-collision shocks have been modelled by Eichler \& Usov (1993). The last four years have seen significant advances, by spectroscopy and highresolution imaging, in our observation of colliding wind binaries (CWBs). Here, we concentrate on observations of the infrared to radio continuum. 


\section{Long-wavelength radiation from WR and OB stellar winds}

The great extension of the WR and $\mathrm{O}$ stellar winds and the $\lambda^{2.1}$-dependence of free-free opacity in this regime give WR and $\mathrm{O}$ continua long-wavelength 'tails' formed by free-free emission. Radio and millimetre observations show powerlaw spectra $\left(S_{\nu} \propto \nu^{\alpha}\right)$ having indices $\alpha \simeq 0.7$, slightly steeper than the $\alpha=0.6$ expected (e.g., Wright \& Barlow 1975) from uniform $\rho \propto r^{-2}$ winds, possibly due to ionization gradients or deviations from a $r^{-2}$-density law (e.g., Abbott et al. 1986; Hogg 1989; Williams et al. 1990b; Leitherer \& Robert 1991). In the infrared, the spectra steepen to $\alpha \simeq 0.9$ as they are formed where the winds are still accelerating and (for different reasons) similar spectral indices are observed in the visible and near-UV (e.g., Morris et al. 1993).

It was evident from the earliest studies that a substantial fraction of WR stars show infrared or radio fluxes significantly higher than those expected from their free-free continua. In the infrared, Planckian spectra peaking near $4 \mu \mathrm{m}$ and having flux levels 1-2 orders of magnitude above the free-free continuum are observed from most of the WC9 and a few WC8 stars and are attributed to persistent formation of circumstellar dust (e.g., Allen et al. 1972; Gehrz \& Hackwell 1974; Williams et al. 1987). The particular significance of this phenomenon is the great difficulty of forming dust in the winds of hot stars, whose radiation fields tend to evaporate grains unless they are shielded in some way or located more than $\sim 50$ AU from the stars. At such distances, the density in a uniform WR wind carrying $\sim 5 \times 10^{-5} \mathrm{M}_{\odot} \mathrm{y}^{-1}$ is too low for molecules and dust to form (Cherchneff \& Tielens 1995).

Surveys of radio emission from hot stars revealed that $25-50 \%$ showed nonthermal emission characterized by flux levels above that expected from the wind, a smaller, often negative $(\alpha \simeq-0.5)$ spectral index and high brightness temperature (e.g., Abbott et al. 1986; Bieging et al. 1989; Scuderi et al. 1998; Chapman et al. 1999). A large fraction ( 70\%, Dougherty, these Proceedings) of the non-thermal sources are observed to be associated with binary systems. The non-thermal component is usually variable, in some cases fading so that only the free-free emission is observed. Note that variability alone is not evidence for non-thermal radiation: witness the changes in the radio flux observed from $\eta$ Carinae as the ionization changes (e.g., White 1999) during its orbit. The non-thermal emission is believed to be synchrotron radiation from electrons accelerated by shocks in wind instabilities in single stars (White 1985), shocks in colliding-wind systems (Eichler \& Usov 1993) or moving current sheets in binaries (Jardine et al. 1996). It is remarkable that we observe the emission at all; the great opacity of stellar winds at $\mathrm{cm}$ wavelengths requires that the electrons are accelerated where the line-of-sight circumstellar extinction is low.

The presence of non-thermal emission undermines the use of radio measurements for determining stellar mass-loss rates (e.g., Abbott et al. 1986), but its negative spectral index ensures that the contribution is negligible at shorter wavelengths, leading Contreras et al. (1996) to use observations at $7 \mathrm{~mm}$ for uncontaminated measures of the stellar wind. Also, modelling of circumstellar dust shells shows that the dust emission falls below that of the stellar wind at $\mathrm{mm}$ wavelengths for even the densest shells, increasing the value of this wavelength region for studying the unadulterated stellar wind emission. 

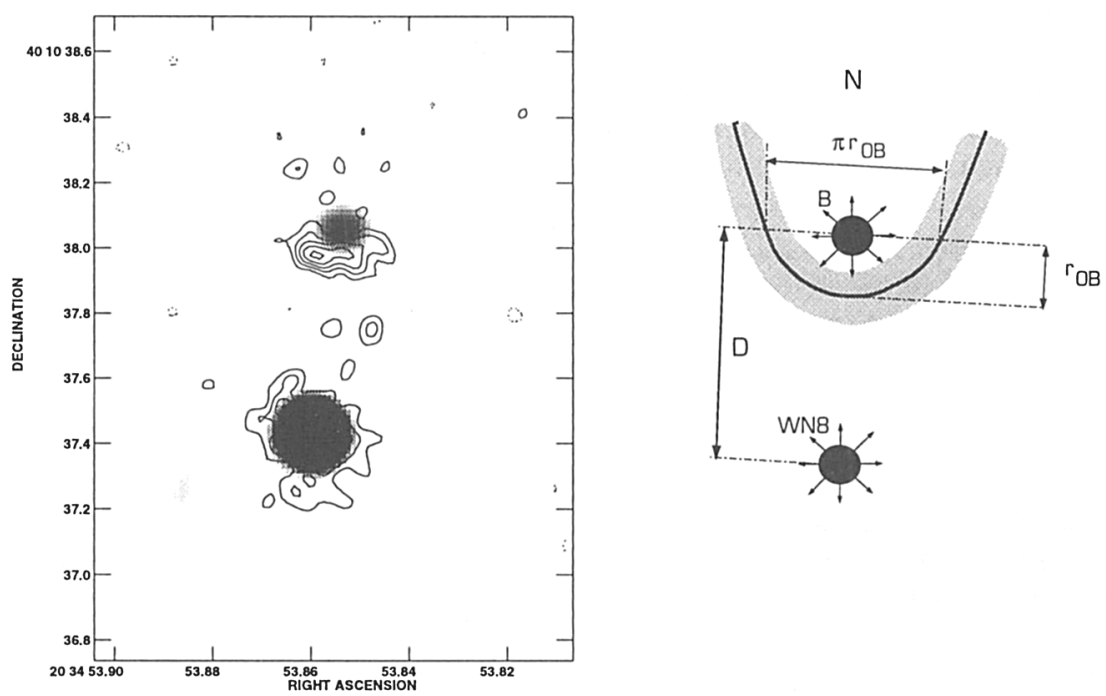

Figure 1. WR 147: overlay of 50-mas resolution MERLIN 5-GHz map (contours) with stellar images and (right) sketch of colliding-wind geometry in which the solid arc is the contact discontinuity.

\section{Radio emission from colliding wind systems}

\subsection{The resolved system WR 147 (AS 431)}

The WN8 system WR 147 (AS 431) has become our archetypal CWB radio source. Its radio emission was first resolved into two components by Moran et al. (1989): one coincident with the optical image of the WN8 star and identified as its free-free emission and the other, $0 ! 6$ to the north, as the possible source of non-thermal emission. From multi-frequency observations, Churchwell et al. (1992) confirmed that WR $147 \mathrm{~N}$ was a non-thermal emitter having a $2-6$-cm spectral index of $\sim-0.5$, compared with a spectral index of $\sim+0.6$ for WR 147S, consistent with free-free emission from the wind of the WN8 star.

New observations with MERLIN after its sensitivity and resolution had been increased by the addition of the Cambridge antenna allowed resolution of both the $\mathrm{N}$ and $\mathrm{S}$ components, showing WR $147 \mathrm{~N}$ to be elongated $\mathrm{E}-\mathrm{W}$, sized $\sim 267 \times 79$ mas (Williams et al. 1997). The same authors discovered (in the near IR) a faint stellar companion to the WN8 star at the same position angle as WR $147 \mathrm{~N}$ but at a separation (635 mas) greater than that of the radio components ( 575 mas). This, and the association of WR 147S with the WN8 star, showed WR $147 \mathrm{~N}$ to be located between the stars, indicating that it arose where the two stellar winds collided. Niemela et al. (1998) observed the faint companion in the optical, measuring the same position angle and separation as in the infrared. Both groups showed that the companion was luminous enough to possess a wind capable of colliding with that of the WN8 star. This occurs where the momenta of the WN8 and OB winds balance, forming a contact discontinuity relatively closer to the OB star owing to its expected lower mass-loss rate. The 
scale of the wind-interaction region is determined by the distance $r_{\mathrm{OB}}$ of the contact discontinuity from the OB star (Eichler \& Usov 1993, whose notation we follow). The contact discontinuity (Fig. 1) has the form of a cap of height $r_{\mathrm{OB}}$ and diameter $\pi r_{\mathrm{OB}}$ centred on the OB star, facing the WN8 star, and a cone facing away from the WN8 star. Shocks form in the winds on either side of this. If the non-thermal emission is produced by electrons accelerated here, we would expect the source to resemble the interaction region both in its proximity to the OB star and in its shape - elongated perpendicular to the line between the components with an aspect ratio $\sim \pi$, depending on the inclination of the system, i.e., the angle $i^{\prime}$ between the axis of symmetry of the contact discontinuity and the plane of the sky. This angle depends on both the orbital inclination and the phase but the latter is not material in such a long-period system. The MERLIN observations fit well in both respects, giving support to the CWB interpretation of the non-thermal emission. From the difference between the radio and average optical and IR separations $(640 \pm 20$ mas $)$, we estimate the projected value of $r_{\mathrm{OB}}$, i.e., $r_{\mathrm{OB}} \cos \left(i^{\prime}\right)=65 \pm 30$ mas. The value of $r_{\mathrm{OB}} \cos \left(i^{\prime}\right)$ determined in this way is very uncertain since it comes from the difference of two larger quantities but it is consistent with the value of $r_{\mathrm{OB}}$ estimated from the width of the source ( $\sim 85$ mas from relating $\pi r_{\mathrm{OB}}$ to the observed width, $\sim 267 \mathrm{mas}$ ). The scale of the system and position of the contact discontinuity are related to the separation of the two stars, $D$, through the ratio of wind momenta, $\eta$, by

$$
r_{\mathrm{OB}}=\frac{\eta^{1 / 2}}{1+\eta^{1 / 2}} D
$$

giving $\eta \simeq 0.013$, a value that is independent of the inclination angle of the system. For spherical winds, $\eta=\left(\dot{M} v_{\infty}\right)_{\mathrm{OB}} /\left(\dot{M} v_{\infty}\right)_{\mathrm{WN}}$. Assuming the wind velocities of the OB and WN8 stars are comparable, we can use $\eta$ and the massloss rate of the WN8 star $\left(\sim 4.6 \times 10^{-5} \mathrm{M}_{\odot} \mathrm{y}^{-1}\right.$, from its radio flux $)$ to estimate that of the OB star: $\sim 6 \times 10^{-7} \mathrm{M}_{\odot} \mathrm{y}^{-1}$.

Recent observations at $3.6 \mathrm{~cm}$ by Contreras \& Rodríguez (1999 and these proceedings) using the VLA (resolution $\sim 200$ mas, but more sensitive than MERLIN for diffuse emission) show the bow shock more clearly. They compared the shape of the shock with the theoretical shape of the contact surface for $\eta=0.011$ to estimate the inclination of the system $\left(i^{\prime} \simeq 45^{\circ} \pm 15^{\circ}\right)$. This assumes that the synchrotron emitting region is symmetric about the contact discontinuity.

The total radio flux from WR 147 has been monitored with the WSRT since 1988 and was found to be variable at both 21 and $6 \mathrm{~cm}$ (van der Hucht et al. 1995). A comparison of VLA 3.6-cm maps observed by Contreras \& Rodríguez (1999) in 1995.55 and 1996.95, shows a significant (25\%) increase in the flux from the WN8 wind component, WR 147S, but no significant change in WR $147 \mathrm{~N}$. Both their maps show an additional emission region to the south of WR 147S from a 'blob' of plasma which moved away from the WN8 star at a projected velocity $\sim 400 \mathrm{~km} \mathrm{~s}^{-1}$ in the interval between the two observations. Also, from a comparison of MERLIN 6-cm maps taken in 1992.48 and 1995.33, Watson et al. (these Proceedings) found morphological changes in both components and that the flux from WR 147S had fallen by $\sim 25 \%$ while the brightness temperature remained the same. The influence of irregularities in the WN8 mass flow into 
the interaction region on the non-thermal emission from WR $147 \mathrm{~N}$ needs to be examined by repeated mapping of the sources. Depending on the inclination angle, the WN8 stellar wind should take $\sim 2$ years to reach the interaction region.

\subsection{Other CWB systems resolved in the radio}

Two other non-thermal radio sources have been shown to be located in windcollision regions. Dougherty et al. (1996) resolved the strong 6-cm radio emission from WR 146 (HM19-3) into two components separated by $116 \pm 14$ mas, one thermal and one non-thermal. They also showed that the optical spectrum of the WR star was composite, suggesting that the WC6 star had an OB companion. From observations at intermediate wavelengths, they identified the thermal radio source with the WR stellar wind. They suggested that WR 146 was a CWB in which the non-thermal emission arose where the WR wind collided with that of the OB star, which they expected be located slightly further away from the WR star. The OB star has since been observed directly in HST images by Niemela et al. (1998), who measured a separation of $168 \pm 31$ mas for the stars. Comparison of this with the separation of the 6 -cm images gave a momentum ratio $\eta \simeq 0.1$ for the system, rather higher than that for WR 147 and suggesting a relatively high mass-loss rate for the OB star. Dougherty \& Williams (these Proceedings) have recently resolved WR 146 into two components using the $V L A$ at $1.3 \mathrm{~cm}$, a wavelength at which the non-thermal contribution was expected to be half that at $6 \mathrm{~cm}$ (for $\alpha=-0.5$ ) whereas the thermal wind emission should be $\sim 2.9$ times higher. They observed two images having the same separation (162 \pm 8 mas) as the optical images and identified them with the wind emission of the two stars. Thus, all three radio components of WR 146 have been observed: the two stellar winds and their interaction. Together with estimating $\eta$ from the geometry (assuming the non-thermal emission to be close to the contact discontinuity), the ratio of wind densities, $\left(\dot{M} / v_{\infty}\right)_{\text {OB }} /\left(\dot{M} / v_{\infty}\right)_{\text {WC }}$, can be derived from the ratio of stellar wind fluxes given knowledge of the wind composition, notably the mean atomic weight, $\mu$, expected to be about four times higher in the chemically evolved wind of the WC6 star than in that of the OB star. These two ratios are independent of distance, so that it should be possible to use them to derive values of $M$ and $v_{\infty}$ for the OB star from the corresponding quantities for the WC6 star. This analysis confirms the relatively high mass-loss rate for the OB star but the model may be too simple (Dougherty \& Williams, these Proceedings).

The total radio flux from WR 146 has been monitored with the WSRT since 1989 , primarily at 6 and $21 \mathrm{~cm}$ where the flux is dominated by that from the non-thermal component, and found to be strongly variable (Setia Gunawan et al. 1997, these Proceedings).

The third CWB system resolved using high-resolution optical and radio observations is the triple system Cyg OB2 No.5 (V729 Cyg). This comprises an O6If+O7If eclipsing binary having a faint companion to the NE, discovered by Herbig (1967) in the optical and in the radio by Abbott et al. (1981). The precise relationship between these images was determined with new astrometry by Contreras et al. (1997). They confirmed the association of the primary radio component with the Of binary using its Hipparcos position and used Hipparcos and CCD imaging to confirm that the optical separation of the NE component 
from the primary exceeded the radio by $0.14 \pm 0.07$. This, and its negative spectral index, identified the radio companion with collision of the Of binary wind with that of an early B-type companion. The radio emission from the binary itself remains enigmatic. It varies with an apparent period $\sim 7$ years between a 'low' state with $\alpha \simeq 0.6$ characteristic of the Of binary wind and a 'high' state with a flatter spectral index indicative of a non-thermal component (Miralles et al. 1994; Contreras et al. 1997), for which a model based on a synchrotron envelope created by periodic production of relativistic electrons has been explored by Miralles et al. An explanation based on a colliding-wind source within the Of binary is unlikely given its very different $(6.6 \mathrm{~d})$ period.

The discovery of a non-thermal source displaced $\sim 3^{\prime \prime}$ from the optical image of WR 39 (Chapman et al. 1999) presents the prospect of another resolved system and further astrometry is needed to explore this.

Two factors help us to observe the non-thermal emission from these relatively wide systems. First, the free-free absorption through the winds of the primary mass-losing stars (WR or Of) to the embedded interaction regions is very small at large distances from the stars. We can relate this to the free-free wind flux from the primary, $S_{\nu}$ at wavelength $\lambda$ by:

$$
\tau \approx 40 \times\left(\frac{S_{\nu} / \mathrm{mJy}}{T / 10^{4} \mathrm{~K}}\right)^{3 / 2} \times\left(\frac{\lambda / \mathrm{cm}}{\theta / \operatorname{mas}}\right)^{3}
$$

where $\theta$ is the angular distance from the star and $T$ the wind temperature, giving, e.g., $\tau \simeq 6 \times 10^{-3}$ at $6 \mathrm{~cm}$ at 575 mas from the WN8 star in WR 147. Secondly, we can be sure that the interaction regions are sufficiently far $\left(\gg 100 R_{\mathrm{OB}}\right.$ ) from the companions for the $\mathrm{OB}$ winds to have been accelerated to the terminal velocities well before they collide and that the WR winds are not slowed by radiative braking before they interact (cf. Gayley et al. 1997).

\subsection{Non-thermal sources suffering variable extinction}

Even if the circumstellar extinction to the non-thermal emission region is not small enough to observe the interaction all the time, in intermediate-sized systems it is sometimes low enough for us to observe non-thermal emission partly absorbed in the WR wind. The best observed example is the WC7+O4-5 binary, WR 140, whose non-thermal emission varies systematically in its orbit (Williams et al. 1990a; White \& Becker 1995). The strong frequency dependence of freefree extinction $\left(\tau_{\nu} \propto \nu^{-2.1}\right)$ allows it and the intrinsic non-thermal emission $\left(I_{\nu}(\mathrm{nT})\right)$ to be determined independently using multi-frequency observations. The variation of $\tau_{\nu}$ to the wind-collision region as the stars move in their orbits enable us to map the circumstellar extinction through the WR wind while any variation in $I_{\nu}(\mathrm{nT})$ gives further information on the wind structure. White \& Becker (1995) observed WR 140 at 2,6 and $20 \mathrm{~cm}$ and found that $I_{\nu}(\mathrm{nT})$ showed a maximum near orbital phase 0.7 . They deduced that the WR wind was flattened into a disk inclined to the orbital plane through which the $\mathrm{O}$ star passed at $\phi \simeq 0.7$. This has still to be modelled quantitatively. Also, Pollock (1998) has developed an alternative model which dispenses with the flattened wind.

A system remarkably similar to WR 140 is WR 125 , whose non-thermal radio flux faded from 1985, to a level consistent with free-free emission (Williams et al. 1992; Contreras et al. 1996). The underlying WR star is very similar to 


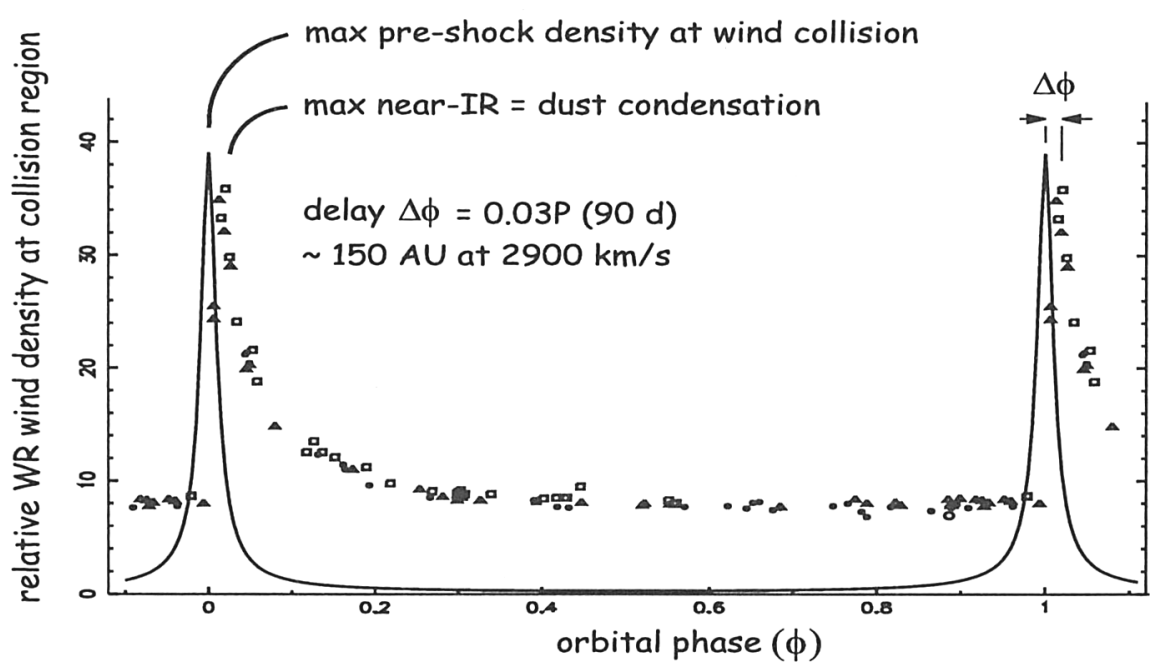

Figure 2. WR 140: comparison of the orbital variation of the WC7 stellar wind density at the wind-collision region (continuous line) with the observed K-band magnitudes (symbols) phased to the orbital elements.

WR 140, both spectroscopically in showing absorption lines suggesting an OB companion, and in that it underwent an episode of dust formation after the nonthermal emission vanished, possibly because the non-thermal source suffered greater extinction as the wind-collision region moved deeper into the WR wind.

A relatively small amount of non-thermal emission has been observed superimposed on the wind spectrum of $\gamma^{2}$ Velorum (Chapman et al. 1999). It is probably significant that the orbital phases at which the observations were made were (fortuitously) those at which the circumstellar extinction to the windcollision region is expected to be smallest (Chapman, private communication).

\section{Dust formation in $\mathrm{CW}$ binaries}

We do not have direct images of colliding wind structures in the IR comparable with those in the radio but the indirect evidence has become ever more compelling and provides a valuable clue to the enigma of dust formation in hostile environments. This process demands density enhancements $\sim 10^{3-4}$ above that of the uniform wind (Cherchneff \& Tielens 1995) and one mechanism which can provide compression on this scale can occur in colliding wind structures if the material flowing along the contact discontinuity can cool efficiently (Usov 1991). Here we ask: what evidence is there that this is happening?

As is often the case in astronomy, the best laboratories are provided by variable objects - such as the archetypal episodic dust-maker WR 140 - and we ask what else changes during the episodes of dust formation. Three of these have been observed, occurring at the same orbital phase, coinciding with periastron passage in the orbit (Williams 1997). This phasing of dust formation to the 
Table 1. Episodic dust-making Wolf-Rayet systems. WR spectrum $P(\mathrm{y})$ dates of IR maxima and notes

\begin{tabular}{llcl}
\hline 19 & WC4+OB & 10.1 & $(1987), 1997-8$ \\
$48 \mathrm{a}$ & WC8-9 & & 1979, also mini eruptions \\
70 & WC9+B0I & & 1989, IR eclipse 1992, SB2 \\
$98 \mathrm{a}$ & WC8-9 & 1.4 & never fades to wind level \\
125 & WC7+O9 & & 1992, non-thermal radio \\
137 & WC7+? & 13.1 & $(1971), 1984,1997$ \\
140 & WC7+O4-5 & 7.94 & SB2, non-thermal radio, X-ray
\end{tabular}

binary orbit provides a crucial clue: the changes in physical conditions in the wind which determine when dust formation occurs must be related to changes with the orbital motion of some long-lived structure in the system. As the WC7 and O4-5 stellar winds in WR 140 collide and compress wind material all the time, we need to enquire what varies round the orbit so as to trigger dust formation for only $\sim 0.02 P$ during periastron passage. Consider the systematic variations in the pre-shock wind density near the interaction region. This region lies where the momenta of the WC7 and O4-5 winds balance and is much closer to the O4-5 star, whose mass-loss rate is $\sim 60$ times smaller than that of the WC7 star. Because the orbit is very eccentric $(e=0.84)$, the separation of the stars and hence the distance of the interaction region to the WC7 star and density of the WC7 stellar wind encountered vary around the orbit. This is especially so around the time of periastron passage: for a very short time, the density of the WC7 stellar wind going into the shock (and being compressed by it) is $\sim 50$ times greater than that during most of the orbit (Fig. 2). The consequent 'spikes' in the pre-shock density appear to be the clock that triggers the dust condensation. The dust cannot condense until the compressed wind material has been carried far enough away from the stars so that the grains are not heated to sublimation by the stellar radiation field: a distance $\sim 150 \mathrm{AU}$. Assuming the compressed material moves with the wind terminal velocity $\sim 2900 \mathrm{~km} \mathrm{~s}^{-1}$, this introduces a delay of $\sim 90$ days between the times of maximum pre-shock wind density (at periastron passage) and maximum dust formation - consistent with the observed phase difference $(\Delta \phi \simeq 0.03 P)$ between maximum pre-shock density and infrared ( $K$ band) maximum (Fig. 2$)$. This provides a very strong link - a 'smoking gun' - between colliding winds and dust formation.

Of the other episodic dust-makers (Table 1), WR 19 showed a short-lived infrared maximum in 1997-98 and appears to have a period of $\sim 10.1$ years (Veen et al. 1998). The spectrum of WR 19 is clearly composite and needs sustained observation over the next decade to look for an orbit and a relation between this and the dust formation episodes to test whether the WR 140 paradigm applies to this system. Single episodes of dust formation have been observed from WR 48a and WR 125 (e.g., Williams 1997), whereas WR 98a (IRAS17380-3031) appears to 'top up' its dust reservoir every $\sim 1.4$ years.

The dust emission from WR 137 (HD 192641) also went through maximum in 1997-98, after rising for some years, suggesting a slower build up of dust 
formation than observed from WR 140. This may be because the eccentricity of WR 137's orbit is apparently much lower than that of WR 140 (Annuk 1995) and a more definitive orbit is now required for modelling the process and examining the CWB connection. The dust-formation period, from two well-observed maxima and the fading from an earlier one, is $\sim 13.1$ years.

Remarkable infrared images of WR 137 observed near maximum in 1997.7 and 1998.4 using HST-NICMOS2 by Marchenko et al. (1999) show very asymmetric dust emission, as one would expect from a CWB, and movement of dust clumps away from the star, presumably with the wind.

\section{Conclusions}

Direct evidence for a colliding-wind origin of non-thermal radio emission from wide $\left(\sim 0^{\prime \prime} .1-1^{\prime \prime}\right)$ luminous binaries has been provided by comparison of highresolution radio and optical imaging. In the infrared, the connection between dust formation and colliding winds has been greatly strengthened by the observation of asymmetric dust formation from one binary and the identification of more candidate CWB dust makers.

Acknowledgments. It is a pleasure to thank Jessica Chapman, Maria Contreras, Augusto Damineli, Bas den Hond, Sean Dougherty, Karel van der Hucht, Sergey Marchenko, Salvo Scuderi, Diah Setia Gunawan, Sarah Watson and Stephen White for providing results in advance of publication and helpful correspondence.

\section{References}

Abbott, D.C., Bieging, J.H., Churchwell, E.B. 1981, ApJ 250, 645

Abbott, D.C., Bieging, J.H., Churchwell, E.B., Torres, A.V. 1986, ApJ 303, 239

Allen, D.A., Harvey, P.M., Swings, J.P. 1972, A\&A 20, 333

Annuk, K. 1995, in: K.A. van der Hucht \& P.M. Williams (eds.), Wolf-Rayet Stars: Binaries, Colliding Winds, Evolution, Proc. IAU Symposium No. 163 (Dordrecht: Kluwer), p. 231

Bieging, J.H., Abbott, D.C., Churchwell, E.B. 1989, ApJ 340, 518

Chapman, J.M., Leitherer, C., Koribalski, B., Bouter, R., Storey, M. 1999, ApJ in press

Cherchneff, I., Tielens, A.G.G.M. 1995, in: K.A. van der Hucht \& P.M. Williams (eds.), Wolf-Rayet Stars: Binaries, Colliding Winds, Evolution, Proc. IAU Symposium No. 163 (Dordrecht: Kluwer), p. 346

Chlebowski, T. 1989, ApJ 342, 1091

Churchwell, E., Bieging, J.H., van der Hucht, K.A., Williams, P.M., Spoelstra, T.A.Th., Abbott, D.C. 1992, ApJ 393, 329

Contreras, M.E., Rodríguez, L.F., Gómez, Y., Velázquez, A. 1996, ApJ 469, 329

Contreras, M.E., Rodríguez, L.F., Tapia, M., Cardini, D., Emanuele, A., Badiali, M., Persi, P. 1997, ApJ 488, L153

Contreras, M.E., Rodríguez, L.F. 1999, ApJ 515, 762

Dougherty, S.M., Williams, P.M., van der Hucht, K.A., Bode, M.F., Davis, R.J. 1996, MNRAS 280, 963

Eichler, D., Usov, V. 1993, ApJ 402, 271 
Gayley, K.G., Owocki, S.P., Cranmer, S.R. 1997, ApJ 475, 786

Gehrz, R.D., Hackwell, J.A. 1974, ApJ 149, 619

Herbig, G.H. 1967, PASP 79, 502

Hogg, D.E. 1989, AJ 98, 282

Jardine, M., Allen, H.R., Pollock, A.M.T. 1996, A\&A 314, 594

Leitherer, C., Robert, C. 1991, ApJ 377, 629

Marchenko, S.V., Moffat, A.F.J., Grosdidier, Y. 1999, in press

Miralles, M.P., Rodriguez, L.F., Tapia, M., Roth, M., Persi, P., Ferrari-Tonolio, M., Curiel, S. 1994, A\&A 282, 547

Moran, J.P., Davis, R.J., Bode, M.F., Taylor, A.R., Spenser, R.E., Argue, A.N., Irwin, M.J., Shanklin, J.D. 1989, Nature 340, 449

Morris, P.W., Brownsberger, K.B., Conti, P.S., Massey, P., Vacca, W. 1993, ApJ 412, 324

Niemela V.S., Shara, M.M., Wallace, D.J., Zurec, D.R., Moffat, A.F.J. 1998, AJ 115, 2047

Prilutskii, O.F., Usov, V.V. 1976, Soviet Ast. 20, 2

Pollock, A.M.T. 1987, ApJ 320, 283

Pollock, A.M.T., 1998, in: L. Kaper \& A.W. Fullerton (eds), Cyclical Variability in Stellar Winds, ESO Astrophysics Symposia Series (Berlin: Springer), p. 110

Scuderi, S., Panagia, N., Stanghellini, C., Trigilio, C., Umana, G., 1998, A\&A 332, 251

Setia Gunawan, D.Y.A., van der Hucht, K.A., de Bruyn, A.G., Williams, P.M. 1996, in: J.M. Vreux et al. (eds.), Wolf-Rayet Stars in the Framework of Stellar Evolution, Proc. 33rd Liège Astrophysical Colloquium, (Université de Liège), p. 327

Stevens, I.R., Blondin, J.M., Pollock, A.M.T. 1992, ApJ 386, 265

Usov, V.V. 1991, MNRAS 252, 49

Usov, V.V. 1992, ApJ 389, 635

van der Hucht, K.A., Williams, P.M., Spoelstra, T.A.Th., Swaanenvelt, J.P., 1995, in: in: K.A. van der Hucht \& P.M. Williams (eds.), Wolf-Rayet Stars: Binaries, Colliding Winds, Evolution, Proc. IAU Symposium No. 163 (Dordrecht: Kluwer), p. 559

Veen, P.M., van der Hucht, K.A., Williams, P.M., Catchpole, R.M., Duijsens, M.F.J., Glass, I.S., Setia Gunawan, D.Y.A. 1998, A\&A 339, L45

White, R.L. 1985, ApJ 289, 698

White, R.L., Becker, R.H. 1995, ApJ 289, 698

White, S. 1999, in: B. Wolf, A. Fullerton \& O. Stahl (eds.), Variable and Non-Spherical Winds in Luminous Hot Stars, Lecture Notes in Physics (Berlin: Springer), p. 80

Williams, P.M. 1997, ApSS 251, 321

Williams, P.M., van der Hucht, K.A., Thé, P.S. 1987, A\&A 182, 91

Williams, P.M., van der Hucht, K.A., Pollock, A.M.T., Florkowski, D.R., van der Woerd, H., Wamsteker, W.M. 1990a, MNRAS 243, 662

Williams, P.M., van der Hucht, K.A., Sandell, G., Thé, P.S. 1990b, MNRAS 244, 101

Williams, P.M., van der Hucht, K.A., Bouchet, P., Spoelstra, T.A.Th., Eenens, P.R.J., Geballe, T.R., Kidger, M.R., Churchwell, E. 1992, MNRAS 258, 461

Williams, P.M., Dougherty, S.M., Davis, R.J., van der Hucht, K.A., Bode, M.F., Setia Gunawan, D.Y.A. 1997, MNRAS 289, 10

Wright, A.E., Barlow, M.J. 1975, MNRAS 170, 41 


\section{Discussion}

Dyson: I did not understand why you said dust formation occurs as the gas flows along the contact discontinuity. The pressure (and thus the density) drops off rapidly as the gas moves away from the bow-shock head.

Williams: The density is too low in the undisturbed wind and the temperature too high nearer the stars.

Zhekov: In the case of WR 140 it is very difficult to form dust in the interaction region, since it is adiabatic and a temperature of $10^{5} \mathrm{~K}$ can be found very far from the axis of symmetry $(D \ll 150 \mathrm{AU})$. One possibility to increase the cooling, is to consider thermal conductivity. In this case the temperature in the interaction region will be lower and the density higher. But since WR 140 is a non-thermal radio source, a magnetic field will be also present, thus, the thermal conduction effects will be extremely reduced. Therefore, we can expect that WR 140 has an adiabatic interaction region, and we cannot form dust in it.

Williams: It happens every 7.94 years.

Dopita: Once dust starts to form, the very high opacity of the dust will produce very large radiative pressure effects, which will provide dynamical effects and compression in the dust-forming layer. This should help keep the density high, and allow further dust formation.

Melnick: Is it possible to use the radio observations of colliding winds to infer something about WR stellar rotation through the anisotropy of their winds?

Williams: In some cases, like WR 140 , it is possible to use the non-thermal emission as a probe of the wind of the WR star, if the non-thermal source suffers variable extinction caused by change in geometry as the stars move in their orbits. This could give the shape of the WR wind, but that is all we can get.

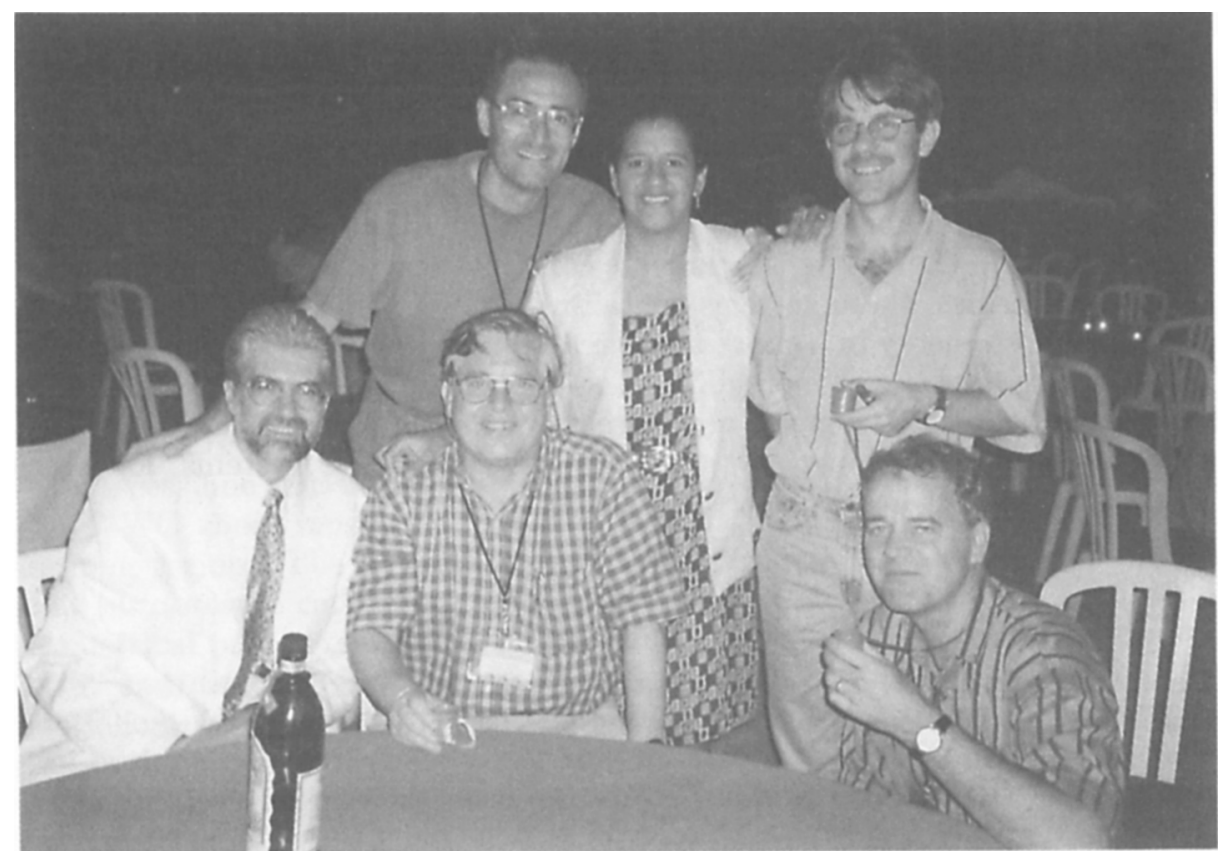

